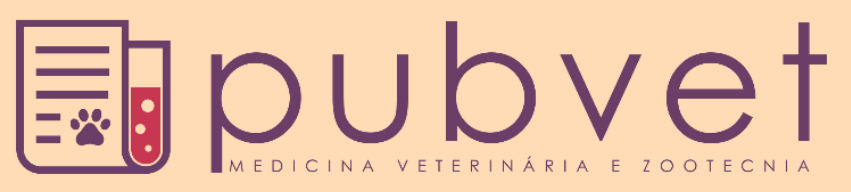

https://doi.org/10.31533/pubvet.v15n12a981.1-7

\title{
Determinação dos parâmetros radiográficos da silhueta cardíaca em cães de raças braquicefálicas clinicamente normais
}

\author{
Edivaldo José Barboza Filho ${ }^{1 *}$, Jackson Farias ${ }^{2}$, Rodrigo Lima Carneiro ${ }^{3}$, Ana Manuella \\ Souza de Babo ${ }^{4} \bullet$
}

${ }^{I}$ Acadêmico da Faculdade Regional da Bahia, curso de Medicina Veterinária. Barreiras -BA Brasil.

${ }^{2}$ Acadêmico da Universidade Estadual da Bahia, curso de Medicina Veterinária. Barreiras -BA Brasil.

${ }^{3}$ Professor da Universidade Estadual da Bahia, Departamento de Medicina Veterinária. Barreiras-BA Brasil.

${ }^{4}$ Professora da Faculdade Regional da Bahia, Departamento de Medicina Veterinária. Barreiras-BA Brasil.

*Autor para correspondência, E-mail: jr-barboza@hotmail.com

\begin{abstract}
Resumo. O objetivo desse estudo foi determinar o valor de VHS em cães de raças braquicefálicas clinicamente normais com objetivo de determinar parâmetros de normalidades para raças com essa característica. O método Vertebral Heart Size (VHS) efetua a mensuração do tamanho cardíaco comparando as vertebras torácicas a fim de auxiliar em diagnostico de insuficiência cardíacas e cardiomegalias. A radiografia de tórax possui uma importância na clínica médica de pequenos animais, trazendo informações para avaliações de órgãos intratorácicos, assim como a capacidade de efetuar avaliação da silhueta cardíaca. As radiografias foram avaliadas de acordo o proposto por Buchanan \& Bücheler (1995), mensurando a silhueta cardíaca e profundidade, e largura do tórax. Os valores médios obtidos para VHS foram de 9,57 $\pm 059 \mathrm{v}$, sendo o menor valor mensurado de $8,5 \mathrm{v}$ e maior $10,5 \mathrm{v}$. A especificação averiguou valores divergentes quando comparado com o estudo dos autores. Confirmando assim, a necessidade de determinação de valores de VHS específicos para raças com características de braquicefalia.
\end{abstract}

Palavras-chave: Coração, radiografia, silhueta cardíaca, VHS

\section{Determination of radiographic parameters of cardiac silhouette in dogs of clinically normal brachycephalic}

\begin{abstract}
The aim of this study was to determine the value of ESR in dogs of clinically normal brachycephalic breeds in order to determine normality parameters for breeds with this characteristic. The Vertebral Heart Size (VHS) method measures heart size comparing thoracic vertebrae in order to help diagnose heart failure and cardiomegaly. Chest radiography has an importance in the medical clinic of small animals, providing information for the evaluation of intrathoracic organs, as well as the ability to assess the cardiac silhouette. The radiographs were evaluated as proposed by Buchanan \& Bücheler (1995), measuring the cardiac silhouette and depth and width of the chest. The mean values obtained for ESR were $9.57 \pm 059 \mathrm{v}$, with the lowest measured value being $8.5 \mathrm{v}$ and the highest $10.5 \mathrm{v}$. The specification found divergent values when compared to the authors' study. Thus, confirming the need to determine specific ESR values or races with characteristics of brachycephaly.
\end{abstract}

Keywords: Heart, radiography, cardiac silhouette, VHS

\section{Introdução}

O presente trabalho traz a determinação de parâmetros radiográficos da silhueta cardíaca em cães de raças braquicefálicas clinicamente normais sob a hipótese de que cães braquicefálicos podem ter valores 
de VHS (vertebral heart size) que divergem do encontrado pelos autores Buchanan \& Bücheler (1995) em seus estudos e serem, ainda assim, clinicamente normais.

O objetivo principal foi determinar o valor de VHS e características subjetivas da silhueta cardíaca radiográfica, em cães de raças braquicefálicas, amplamente difundidas em nosso meio, e adicionalmente buscar características de normalidade para servir de parâmetros radiográficos em cães das raças: Pug, Shihtzu, Lhasa Apso, Pequinês, Boxer e Buldogue Francês.

$\mathrm{O}$ assunto se justifica pela prevalência das doenças valvares, principalmente as que acometem as válvulas mitrais, que são alterações cárdicas comumente observadas em cães de pequeno porte tais como os das raças braquicefálicas. Os métodos de mensuração visam avaliar de forma quantitativa o tamanho do coração com finalidade de aumentar a acurácia e diminuir a subjetividade do estudo radiográfico da silhueta cardíaca.

Esforços têm sido feitos objetivando adaptar métodos de mensurações para animais, embora relativamente aos cães não exista, de maneira bem definida, uma configuração da silhueta cardíaca considerada normal para todas as raças.

\section{Material e métodos}

Esse trabalho foi realizado em duas clínicas veterinárias, localizadas em Barreiras-BA. Avaliou-se imagens radiográficas com intuito de determinar o valor médio da silhueta cardíaca de cães braquicefálicos clinicamente normais, escolhidos por um médico veterinário com base em avaliação física, eletrocardiografia e exames de hemograma completo, mensuração sérica de ureia, creatinina, fosfatase alcalina e gama glutamil transpeptidase no período de novembro de 2018 a março de 2021.

Foram avaliados 50 cães, sendo 31 da raça Shih-Tzu (62\%), sete Pugs (14\%), três Boxers (6\%), três Lhasa Apso (6\%), três Buldogue Frances (6\%), três Pequinês (\%), destes 22 machos (44\%) e 28 fêmeas (56\%) com pesos variando de $1,8 \mathrm{~kg}$ até $6,0 \mathrm{~kg}$.

Os animais selecionados passaram por radiografia digital, posicionados em projeções decúbito lateral e ventrodorsal, escolhendo sempre o momento de inspiração máxima. As avaliações radiográficas contemplaram todo o tórax, com intuito de excluir qualquer anormalidade presente nos pacientes. Logo após a avaliação da silhueta cardíaca, utilizou-se o método de VHS citada por Buchanan \& Bücheler (1995). Na mensuração, usou-se de régua para medir os eixos cardíacos, e com os resultados, as imagens foram passadas para o programa digital que realiza a medição automática, a fim de conferir e comparar os valores adquiridos.

A técnica consististe em mensurar o ângulo longo e curto dos eixos cardíacos, obtendo assim seus valores em centímetros, logo após é posicionado essa distância na quarta vertebra torácica (T4) do animal, convertendo assim os centímetros para unidade de vertebras, fazendo isto com os dois eixos (Figuras 1 e 2). A soma dos eixos convertidos em vértebras é somada obtendo assim o valor do VHS. Na projeção ventrodorsal, a forma de avaliação é similar, e as medidas obtidas são mensuradas com a imagem do animal em decúbito lateral.

Os valores alcançados, foram avaliados de forma estatística descritiva, para o cálculo de desvio padrão e valor médio e efetuou-se uma comparação dos valores obtidos no posicionamento ventrodorsal e laterolateral. Ato contínuo, as mensurações encontradas foram comparadas com estudo de Buchanan \& Bücheler (1995) o qual avaliou 100 animais de várias raças.

Para uma avaliação do tórax, foram também efetuados mensuração da profundidade (Pt) torácica na projeção laterolateral, medida a partir da borda cranial do processo xifoide e a borda ventral da coluna vertebral, enquanto a largura torácica (Lt) foi calculada com a distância entre as bordas mediais das oitavas costelas, junto às suas curvaturas mais laterais obtendo assim a qualidade torácica, que de acordo com Buchanan \& Bücheler (1995) se a relação Pt:Lt evidenciar valores maiores ou iguais a 1,25 é considerado profundo, e largo caso a relação for menor ou igual a 0,75 . 


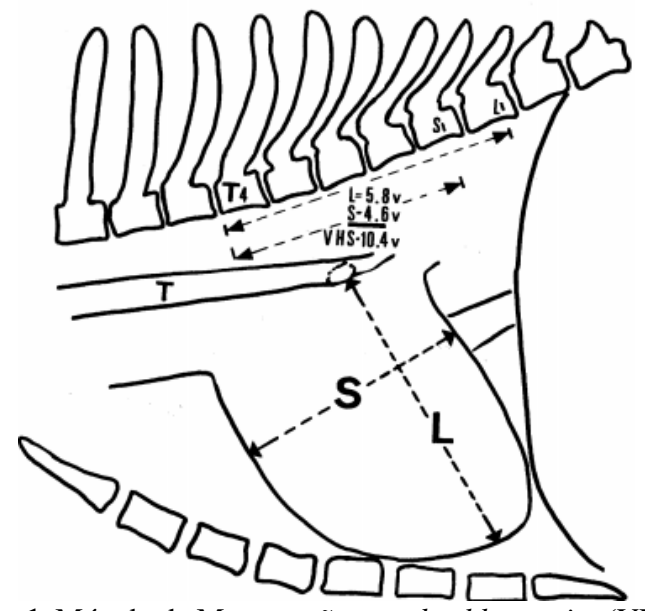

Figura 1. Método de Mensuração vertebral heart size (VHS) na projeção laterolaeral, sendo L o eixo maior e S o eixo menor, $\mathrm{T}$ traqueia e $\mathrm{T} 4$ a quarta vértebra torácica. Fonte: Buchanan \& Bücheler (1995).

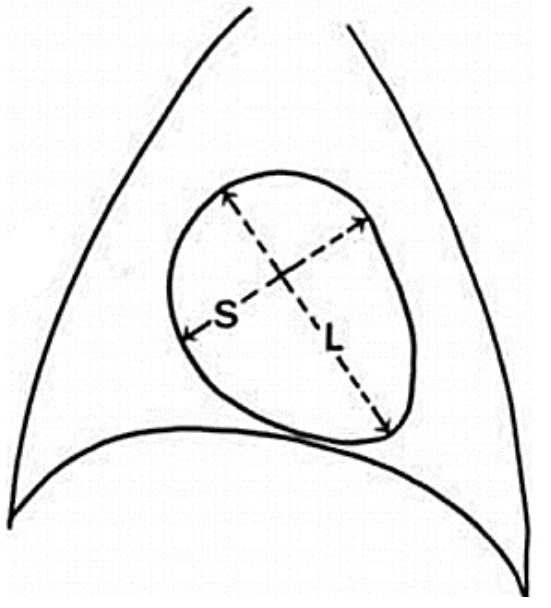

Figura 2. Método de Mensuração vertebral heart size (VHS) na projeção ventrodorsal ou dorsoventral, sendo L o eixo maior e S o eixo menor. Fonte: Buchanan \& Bücheler (1995).

\section{Resultados e discussão}

Os resultados da presente pesquisa demonstraram que dos 50 animais que foram avaliados, tiveram o VHS com valor médio $9,57 \mathrm{v} \pm 0,59 \mathrm{v}$ diferentemente dos valores relatados por Buchanan \& Bücheler (1995) que encontraram em seu estudo, que avaliou 100 animais de raças diferentes, os valores de 9,70v $\pm 0,50$. Além disso, Buchanan \& Bücheler (1995) determinaram que $98 \%$ dos animais avaliados tiveram o VHS mensurado entre 8.5 a 10,6v, ficando estabelecido os valores máximos das dimensões cardíacas sendo de $\leq 10,5 \mathrm{v}$. Em nosso estudo, obtivemos similaridades quanto a esses valores, tendo avaliado o menor VHS de 8,5 e o maior de $10,5 \mathrm{v}$.

As raças analisadas tiveram como padrão a braqueocefalia, sendo avaliado os valores médios do VHS individualmente de acordo com a quantidade encontrada no estudo. A raça Shih-Tzu apresentou valor médio de 9,5v $\pm 0,61 \mathrm{v}$, os Pugs, 9,6v $\pm 0,40 \mathrm{v}$, os Lhasa Apso, 9,66v $\pm 0,76 \mathrm{v}$, os Pequinês 9,16v \pm 0,28v, os Boxers, 9,66v \pm 0,28v e os Buldogues Franceses, 10,00v $\pm 0,86 \mathrm{v}$ (Tabela 1).

Diversos estudos já demonstraram a necessidade de avaliar os diferentes padrões de raças, visto que a conformação de cada uma pode alterar os valores estabelecidos por Buchanan \& Bücheler (1995) sem apresentar anormalidade clínica. Isso foi observado no estudo de Starling (2017) que avaliou a raça Buldogue Campeiro e teve como resultado do VHS médio $11,89 \mathrm{v} \pm 0,70 \mathrm{v}$ demonstrando valores superiores aos estabelecidos por estudos anteriores.

Diferente do que constatado por Sánchez et al. (2012) que afirmaram que fêmeas apresentaram valores menores quando comparado aos do macho, nesta pesquisa as fêmeas apresentaram valores médio de $9,62 \mathrm{v} \pm 0,64 \mathrm{v}$ de VHS enquanto os machos tiveram média de 9,50v $\pm 0,53 \mathrm{v}$ (Tabela 2).

Quanto a conformação torácica, o estudo apresentou 28 animais, classificados como intermediários, 21 animais como largos e apenas 1 como profundo, havendo a relação $\mathrm{P} / \mathrm{L}$ obtendo a média de $0,80 \pm 0,12$.

O coeficiente de correlação de Pearson (r) foi calculado com intuito de estabelecer uma correlação entre a profundidade, largura e a relação PL do tórax quanto o VHS dos animais avaliados assim como a significância $(\mathrm{P} \leq 0,05)$ dos resultados encontrados. Os resultados foram uma correlação positiva quanto a relações da largura e da profundidade sendo $r \leq 0,09 \leq 0,17$ e p $0,024 \leq 0,026$ (Figura 3 e 4 ), e VHS e negativa quanto a relação $\mathrm{P} / \mathrm{L}$ com resultado de $\mathrm{r}=-0,13$ e $\mathrm{P}=0,011$ (Figura 5). Assim como Buchanan \& Bücheler (1995) não foi encontrado correlação significativa da qualidade torácica e o VHS como demonstrados nos gráficos 1,2 e 3.

Azevedo (2018) cita que as patologias cardíacas possuem um acometimento de alta prevalência, sendo que as doenças cárdicas adquiridas se sobressaem em relação as malformações congênitas e genéticas. Castro et al. (2009) em seu estudo avaliou 750 animais com alterações cardíacas, onde foi diagnosticado doença valvular adquirida com $76,7 \%$, cardiomiopatia dilatada com $9,8 \%$, alterações 
congênitas com $6,8 \%$, hipertrofia do ventrículo esquerdo com $3,2 \%$, hipertensão pulmonar com 1,7\%, Neoplasias com 1,3\% e Efusão pericárdicas com 0,5\%.

Tabela 1. Cães avaliados em relação ao VHS, profundidade, largura e qualidade torácica (P/L) no período de 2018 a 2021.

\begin{tabular}{|c|c|c|c|c|c|}
\hline Raça & VHS & Profundidade & Largura & $\mathrm{P} / \mathrm{L}$ & Tórax \\
\hline$\overline{\text { Shih Tzu }}$ & 9,0 & $11,0 \mathrm{~cm}$ & $15,0 \mathrm{~cm}$ & 0,73 & Largo \\
\hline Shih Tzu & 10,0 & $13,5 \mathrm{~cm}$ & $14 \mathrm{~cm}$ & 0,96 & Intermediário \\
\hline Shih Tzu & 10,0 & $11 \mathrm{~cm}$ & $13,5 \mathrm{~cm}$ & 0,81 & Intermediário \\
\hline Shih Tzu & 10,0 & $11 \mathrm{~cm}$ & $14,5 \mathrm{~cm}$ & 0,75 & Largo \\
\hline Shih Tzu & 9,5 & $8,5 \mathrm{~cm}$ & $10,5 \mathrm{~cm}$ & 0,80 & Intermediário \\
\hline Shih Tzu & 10,0 & $6 \mathrm{~cm}$ & $8,5 \mathrm{~cm}$ & 0,70 & Largo \\
\hline Shih Tzu & 9,5 & $7 \mathrm{~cm}$ & $9,5 \mathrm{~cm}$ & 0,73 & Largo \\
\hline Shih Tzu & 10,5 & $6,5 \mathrm{~cm}$ & $8,5 \mathrm{~cm}$ & 0,76 & Intermediário \\
\hline Shih Tzu & 9,0 & $5,5 \mathrm{~cm}$ & $8,5 \mathrm{~cm}$ & 0,64 & Largo \\
\hline Shih Tzu & 10,0 & $5,5 \mathrm{~cm}$ & $8,5 \mathrm{~cm}$ & 0,64 & Largo \\
\hline Shih Tzu & 9,0 & $9,0 \mathrm{~cm}$ & $11,5 \mathrm{~cm}$ & 0,78 & Intermediário \\
\hline Shih Tzu & 9,0 & $6,5 \mathrm{~cm}$ & $8,5 \mathrm{~cm}$ & 0,76 & Intermediário \\
\hline Shih Tzu & 9,0 & $7 \mathrm{~cm}$ & $9,5 \mathrm{~cm}$ & 0,73 & Largo \\
\hline Shih Tzu & 9,0 & $11,5 \mathrm{~cm}$ & $15 \mathrm{~cm}$ & 0,76 & Intermediário \\
\hline Shih Tzu & 8,5 & $7,5 \mathrm{~cm}$ & $9,5 \mathrm{~cm}$ & 0,78 & Intermediário \\
\hline Shih Tzu & 10,0 & $9,0 \mathrm{~cm}$ & $11,5 \mathrm{~cm}$ & 0,78 & Intermediário \\
\hline Shih Tzu & 9,5 & $8,5 \mathrm{~cm}$ & $11,5 \mathrm{~cm}$ & 0,74 & Largo \\
\hline Shih Tzu & 10,0 & $7,0 \mathrm{~cm}$ & $9,5 \mathrm{~cm}$ & 0,73 & Largo \\
\hline Shih Tzu & 10,5 & $7,0 \mathrm{~cm}$ & $9,5 \mathrm{~cm}$ & 0,73 & Largo \\
\hline Shih Tzu & 8,5 & $7,5 \mathrm{~cm}$ & $9,5 \mathrm{~cm}$ & 0,78 & Intermediário \\
\hline Shih Tzu & 8,5 & $8,5 \mathrm{~cm}$ & $8,5 \mathrm{~cm}$ & 0,76 & Intermediário \\
\hline Shih Tzu & 9,5 & $7,5 \mathrm{~cm}$ & $10,0 \mathrm{~cm}$ & 0,75 & Largo \\
\hline Shih Tzu & 10,0 & $7,0 \mathrm{~cm}$ & $8,0 \mathrm{~cm}$ & 0,87 & Intermediário \\
\hline Shih Tzu & 10,5 & $7,0 \mathrm{~cm}$ & $11,0 \mathrm{~cm}$ & 0,63 & Largo \\
\hline Shih Tzu & 10,0 & $7,0 \mathrm{~cm}$ & $10,0 \mathrm{~cm}$ & 0,70 & Largo \\
\hline Shih Tzu & 8,5 & $8,0 \mathrm{~cm}$ & $10,5 \mathrm{~cm}$ & 0,76 & Intermediário \\
\hline Shih Tzu & 9,5 & $7,5 \mathrm{~cm}$ & $8,5 \mathrm{~cm}$ & 0,88 & Intermediário \\
\hline Shih Tzu & 10,0 & $8,0 \mathrm{~cm}$ & $9,5 \mathrm{~cm}$ & 0,84 & Intermediário \\
\hline Shih Tzu & 9,0 & $6,0 \mathrm{~cm}$ & $7,5 \mathrm{~cm}$ & 0,80 & Intermediário \\
\hline Shih Tzu & 9,5 & $8,5 \mathrm{~cm}$ & $11,5 \mathrm{~cm}$ & 0,74 & Largo \\
\hline Shih Tzu & 9,5 & $9,5 \mathrm{~cm}$ & $13,5 \mathrm{~cm}$ & 0,70 & Largo \\
\hline Pug & 9,5 & $14,5 \mathrm{~cm}$ & $16,5 \mathrm{~cm}$ & 1,13 & Intermediário \\
\hline Pug & 10,0 & $12,0 \mathrm{~cm}$ & $15 \mathrm{~cm}$ & 0,8 & Intermediário \\
\hline Pug & 9,5 & $9,0 \mathrm{~cm}$ & $7 \mathrm{~cm}$ & 1,28 & Profundo \\
\hline Pug & 10 & $13,5 \mathrm{~cm}$ & $18 \mathrm{~cm}$ & 0,75 & Largo \\
\hline Pug & 10 & $13,5 \mathrm{~cm}$ & $18 \mathrm{~cm}$ & 0,75 & Largo \\
\hline Pug & 9,0 & $8,5 \mathrm{~cm}$ & $11,5 \mathrm{~cm}$ & 0,73 & Largo \\
\hline Pug & 10,5 & $9 \mathrm{~cm}$ & $12 \mathrm{~cm}$ & 0,75 & Largo \\
\hline Lhasa Apso & 9,5 & $12,5 \mathrm{~cm}$ & $13,5 \mathrm{~cm}$ & 0,92 & Intermediário \\
\hline Lhasa Apso & 9,0 & $10,5 \mathrm{~cm}$ & $10,5 \mathrm{~cm}$ & 1,00 & Intermediário \\
\hline Lhasa Apso & 10,5 & $9,0 \mathrm{~cm}$ & $11,0 \mathrm{~cm}$ & 0,81 & Intermediário \\
\hline Pequinês & 9,0 & $11,0 \mathrm{~cm}$ & $12,5 \mathrm{~cm}$ & 0,96 & Intermediário \\
\hline Pequinês & 9,0 & $10,5 \mathrm{~cm}$ & 10,0 & 1,05 & Intermediário \\
\hline Pequinês & 9,5 & $11,0 \mathrm{~cm}$ & $11,0 \mathrm{~cm}$ & 1,00 & Intermediário \\
\hline Boxer & 10,0 & $18,0 \mathrm{~cm}$ & $17,5 \mathrm{~cm}$ & 1,02 & Intermediário \\
\hline Boxer & 9,5 & $14,5 \mathrm{~cm}$ & $18,5 \mathrm{~cm}$ & 0,78 & Intermediário \\
\hline Boxer & 9,5 & $9,5 \mathrm{~cm}$ & $11,0 \mathrm{~cm}$ & 0,86 & Intermediário \\
\hline Buldogue Francês & 10,5 & $9,5 \mathrm{~cm}$ & $13,5 \mathrm{~cm}$ & 0,70 & Largo \\
\hline Buldogue Francês & 10,5 & $8,0 \mathrm{~cm}$ & $11,5 \mathrm{~cm}$ & 0,69 & Largo \\
\hline Buldogue Francês & 9,0 & $10,5 \mathrm{~cm}$ & $13,5 \mathrm{~cm}$ & 0,77 & Intermediário \\
\hline
\end{tabular}

No presente estudo, os animais avaliados passaram por uma triagem e seleção para determinar higidez, o qual um médico veterinário efetuou avaliação física, eletrocardiografia e exames laboratoriais para serem efetuadas as radiografias torácicas para mensuração do VHS.

As radiografias foram efetuadas em projeção laterolateral direita para uniformizar a avaliação, visto que Buchanan \& Bücheler (1995) em suas mensurações de VHS não encontrou consideráveis alterações de valores em outras projeções como laterolateral esquerda e ventrodorsal. Nesse estudo não foi identificado distinção de tamanho cardíaco quanto ao sexo e tamanho dos animais, o que é fundamentado pela avaliação de Cardoso et al. (2011) que demonstraram em seu estudo que tamanhos e sexos não 
possui importância quando a mensuração de VHS, e quanto possíveis alterações de VHS em posicionamento diferente se deve ao ciclo cardíaco e fases respiratórias

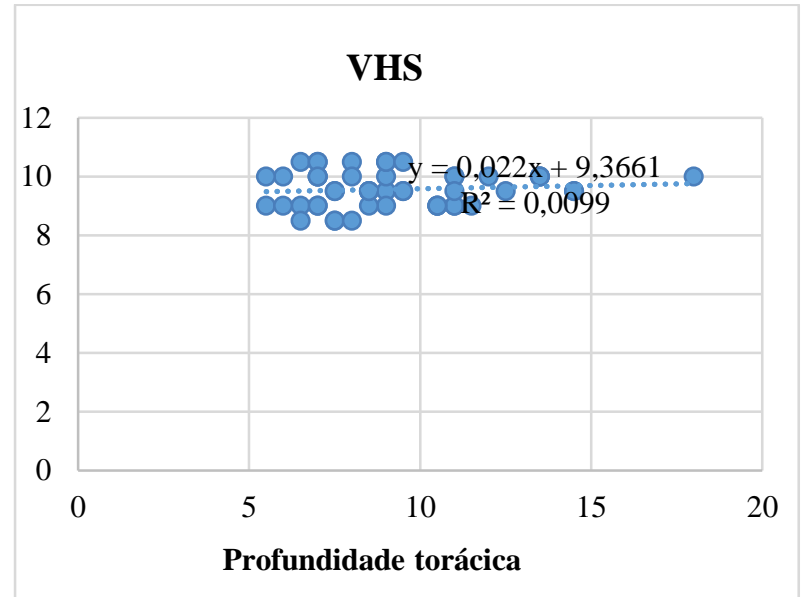

Figura 3. Correlação do VHS com a profundidade torácica $\left(\mathrm{R}^{2}=\right.$ Coeficiente de relação/ $\mathrm{y}=$ Equação de determinação).

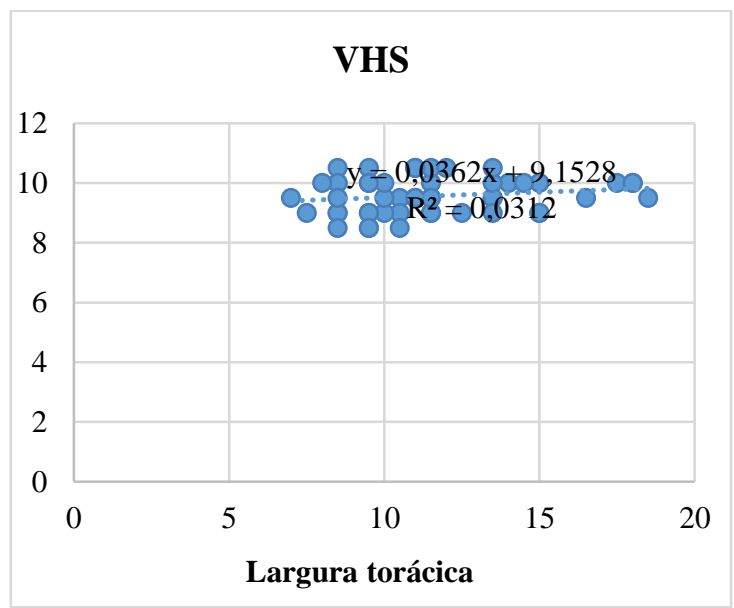

Figura 4. Correlação do VHS com a largura torácica $\left(\mathrm{R}^{2}=\right.$ Coeficiente de relação/ $y=$ Equação de determinação).

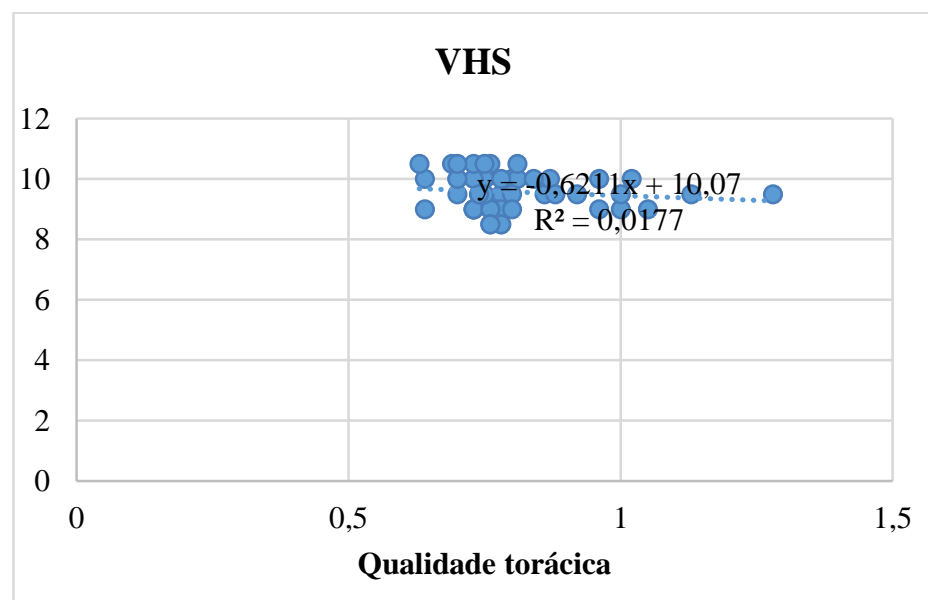

Figura 5. Correlação do VHS com a qualidade torácica $\left(\mathrm{R}^{2}=\right.$ Coeficiente de relação/ y = Equação de determinação).

Em relação a faixa etária, o estudo se atentou a animais entre um a seis anos, sem patologias, avaliada por exames físicos, exames laboratoriais. A avaliação se teve a essa faixa de idade para evitar uma possível alteração do VHS devido ao tamanho e desenvolvimentos dos cães não adultos, apesar que Sleeper \& Buchanan (2001) ao avaliar no seu estudo o VHS de cães filhotes e adultos, não encontraram valores divergentes de animais com três aos 36 meses.

Os 50 cães dessa pesquisa, eram braquiocefálicos, hígidos, com idade que varia entre dois a nove anos que constataram valores de VHS entre 8,5 a 10,5 vertébras. As raças avaliadas foram Pug, Boxer, Shih tzu, Lhasa apso, Pequinês e Buldogues Francês. O período de avaliação ocorreu num período de 15 meses com animais de diferentes raças com padrão de braquicefalia o que torna o estudo uma avaliação de extrema valia, visto que Buchanan \& Bücheler (1995) determinaram que o padrão e raça de animais pode ter uma influência no VHS dos animais, tornando importante a padronização da mensuração média para estes padrões de raças.

Foi avaliado nessa pesquisa a relação de profundidade/largura torácica, com a intenção de demonstrar as possíveis diferenças quanto a conformação do tórax da mesma raça, classificando-os em profundo, largo ou intermediário. A avaliação da profundidade seguiu o padrão citado por Starling (2017) em posição de laterolateral (Figura 6) mensurada da margem cranial do processo xifoide até a borda da ventral do processo vertebral, e a largura torácica, em projeção ventrodorsal, calculada da distância da borda mediais da oitava costela (Figura 7). 
Quanto a relação dos tamanhos (Starling, 2017) cita: "Cães cuja relação P/L excede 1,25 são considerados como tórax profundo, enquanto valores inferiores a 0,75 o tórax é considerado largo. Medidas entre estes valores caracterizam o tórax com conformação intermediária".

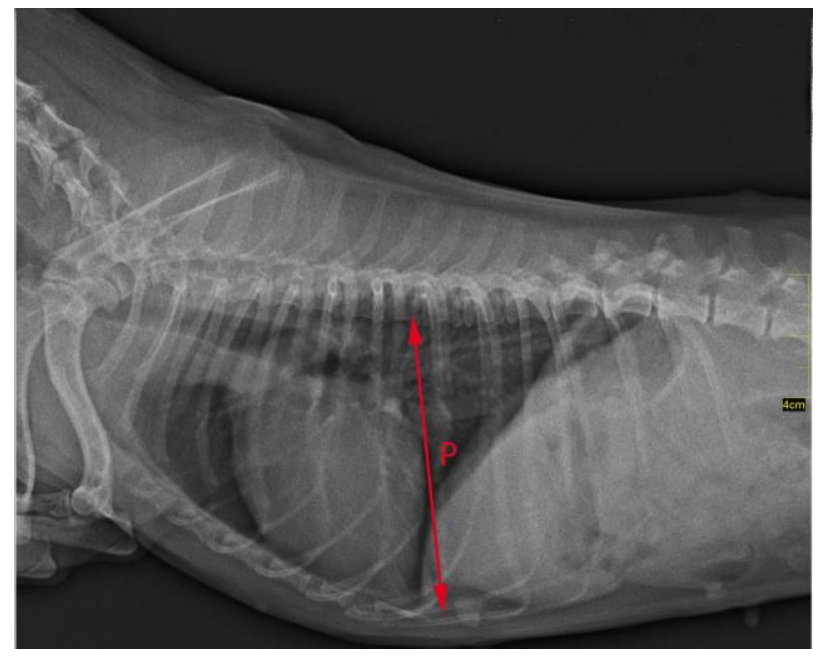

Figura 6. Método de Mensuração da Profundidade Torácica (P) na projeção laterolateral.

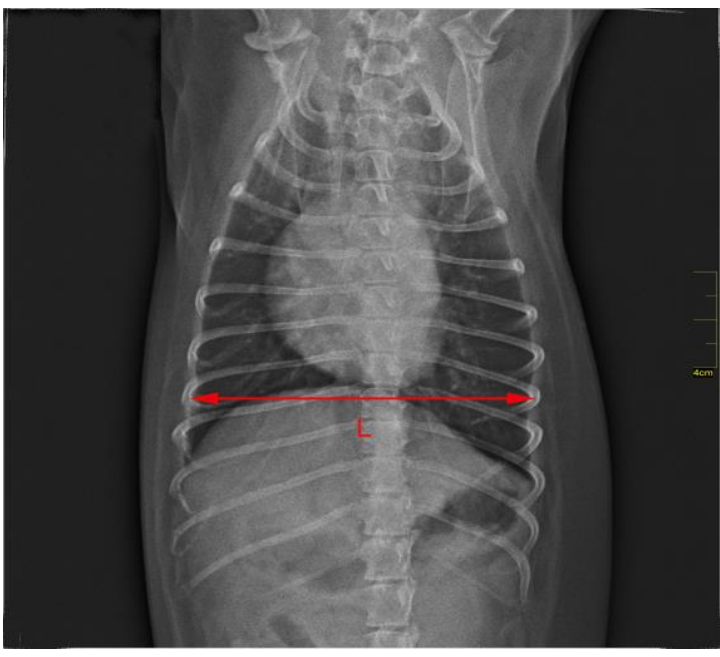

Figura 7. Método de Mensuração da Largura Torácica (L) na projeção ventrodorsal.

Na avaliação da qualidade torácica, 28 animais (56\%) foram classificados em tórax intermediário, $21(42 \%)$ como largos e apenas um (2\%) apresentou o tórax profundo. Ao efetuar uma comparação com estudos de outras raças, como no estudo de (Starling, 2017) que animais da raça buldogue campeiro, raça essa também classificados como braquicefálico, a maioria dos animais também possuía a conformação torácica como intermediário, e alguns largo, porém não apresentou nenhum animal com tórax profundo, o que nos leva a concluir que cães com tórax largo é uma conformação rara. Apesar dos tipos de tórax apresentado por diversos animais, assim como no estudo do Buchanan \& Bücheler (1995) não foi correlacionado nenhuma diferença que caracterize que o tipo de conformação torácica influencie no VHS dos animais.

As dimensões do VHS dos animais pesquisados nesse estudo, ficou no limite encontrado por Buchanan \& Bücheler (1995), nos valores entre 8,5 a 10,5v, quando comparado ao estudo de Starling (2017) seus resultados apresentou valores diferentes, tendo em média em animais adultos VHS de 11,84 $\pm 0,48 \mathrm{v}$, o que de acordo a autora, pode ser explicado devido a conformação estrutural do porte da raça avaliada, que possui uma alta condição atlética, e grande massa muscular, com pesos variando de 35 e $45 \mathrm{~kg}$, que por necessitar um debito cardíaco alto apresenta maiores índices de VHS, devido as altas demandas fisiológicas. Visto isso, pode explicar que em nosso estudo, os pacientes são classificados em porte pequeno, baixo índice de peso corporal e massa muscular, com pesos variando de um a seis $\mathrm{kg}$, apresentando necessidades menores quando comparados a cães de grande porte.

No estudo de Swetha (2020) raças braquiocefálicas apresentaram médias maiores, sendo os valores de VHS maiores do que 9,7 $\pm 0,5$. Observou que na raça Pug as mensurações eram mais altas, 10,7 \pm 0,9 enquanto em nosso estudo a média para raça Pug se manteve no limite esperado 9,6 $\pm 0,40$.

\section{Referências bibliográficas}

Azevedo, G. M. (2018). AVeterbral Heart Scale (VHS) e Índice Cardiotorácico (ICT) e sua relação com A Fração e Ejeção e de Encurtamento em cães Poodle com função cardíaca preservada. Universidade Federal do Piaú́.

Buchanan, J. W., \& Bücheler, J. (1995). Vertebral scale system to measure canine heart size in radiographs. Journal-American Veterinary Medical Association, 206, 194-199. https://doi.org/10.1016/S0195-56169(00)50027-8.

Cardoso, M. J. L., Caludino, J. L., \& Melussi, M. (2011). Mensuração do tamanho cardíaco pelo método VHS (vertebral heart size) em cães sadios da raça American pit bull terrier. Ciência Rural, 41, $127-$ 131. https://doi.org/10.1590/S0103-84782011000100020. 
Castro, M. G., Veado, J. C. C., Silva, E. F., \& Araújo, R. B. (2009). Estudo retrospectivo ecodopplercardiográfico das principais cardiopatias diagnosticadas em cães. Arquivo Brasileiro de Medicina Veterinária e Zootecnia, 61(5), 1238-1241. https://doi.org/10.1590/S010209352009000500032.

Sánchez, X., Prandi, D., Badiella, L., Vázquez, A., Llabrés-Díaz, F., Bussadori, C., \& Domènech, O. (2012). A new method of computing the vertebral heart scale by means of direct standardisation. Journal of Small Animal Practice, 53(11), 641-645. https://doi.org/10.1111/j.17485827.2012.01288.x.

Sleeper, M. M., \& Buchanan, J. W. (2001). Vertebral scale system to measure heart size in growing puppies. Journal of the American Veterinary Medical Association, 219(1), 57-59. https://doi.org/10.2460/javma.2001.219.57.

Starling, J. E. M. de O. (2017). Padronização do método VHS (Vertebral Heart Size) em cães hígidos da raça Buldogue Campeiro. Universidade de Brasília.

Swetha, P. (2020). Computed radiographic studies on size of the heart based on vertebral heart score in dogs at different age groups. Indian Journal of Veterinary Anatomy, 32(2), 15-18.

Histórico do artigo:

Recebido: 18 de agosto de 2021

Aprovado: 28 de setembro de 2021

Disponível online: 9 de novembro de 2021
Licenciamento: Este artigo é publicado na modalidade Acesso Aberto sob a licença Creative Commons Atribuição 4.0 (CC-BY 4.0), a qual permite uso irrestrito, distribuição, reprodução em qualquer meio, desde que o autor e a fonte sejam devidamente creditados. 